\title{
Leuconostoc citreum
}

National Cancer Institute

\section{Source}

National Cancer Institute. Leuconostoc citreum. NCI Thesaurus. Code C86495.

A species of facultatively anaerobic, gram positive, cocci shaped bacteria in the phylum Firmicutes. This species is nonmotile, catalase and oxidase negative, produces acid from arabinose and trehalose and hydrolyzes esculin. L. citreum is usually nonpathogenic and is commonly found in farming soil; it is used as a starter culture for the fermentation of kimchi. 